\title{
Conversion Disorder in a Pharmacogenomics, Polypharmacy Patient: Case Report
}

Michael J. Schuh, PharmD, MBA, FAPhA; Sheena Crosby, PharmD, BCGP

Mayo Clinic, Jacksonville, Florida

\begin{abstract}
Background: Conversion disorder (CD) is a relatively common psychiatric disorder likely encountered by clinical pharmacists but probably not easily identified by pharmacists.

Case Summary: This is a patient case where a patient with a tremor was referred to the pharmacist led, polypharmacy, pharmacogenomics (PGX) service to rule out a PGx cause due to medication metabolism. No pharmacologic or PGx cause was found for the tremor which helped support and confirm a diagnosis of $C D$.

Practice Implications: By working collaboratively with psychiatrists, neurologists, physical medicine colleagues, clinical pharmacists may add value to patient care by assisting with diagnoses and appropriate treatment.
\end{abstract}

Keywords: conversion disorder, case report, pharmacogenomics, polypharmacy

\section{Background}

Conversion disorder (CD) constitutes 1-9\% of psychiatric patients and is only misdiagnosed in $4 \%$ of these patients. ${ }^{1}$ Therefore it is relatively common and accurately diagnosed in psychiatry. CD may present as a neurologic disorder but have no indication of nerve damage or alteration in normal function. $20-25 \%$ of patients in a hospital setting have symptoms of conversion and $5 \%$ meet criteria for full CD. ${ }^{2}$ Since it is a relatively common psychiatric disorder, it is likely encountered by ambulatory, clinical pharmacists but probably not as easily recognized. Since pharmacists are medication centered, looking for medication related problems (MRPs), they may fail to recognize patients with conversion disorder because they do not have a MRP. The patient may be taking medications for symptoms but they may have little effect as treatment because the root cause is a psychiatric disorder. ${ }^{3}$ Therefore $C D$ is common and widely recognized in psychiatry, though possibly less so in pharmacy because pharmacist training does not lend itself to psychiatric conditions that are not directly drug related. One may easily confuse CD with somatic symptom disorder (SSD) since they are very close in presentation but there are differences with regard to the American Psychiatric Association's Diagnostic and Statistical Manual of Mental Disorders, $5^{\text {th }}$ Edition (DSM-5). ${ }^{4}$ Primary differences between CD and SSD are that CD is a subset of SSD and confined to the voluntary central nervous system with symptoms manifesting as neurologic symptoms that do not follow known neurologic damage patterns. SSD is a more general syndrome that may not necessarily manifest as a specific neurological symptom. ${ }^{5}$ (Table I) CD symptoms are difficult to quantify with objective measures via neurologic exam or laboratory tests, but are very real. In addition, CD patients normally see multiple primary and

Corresponding author: Michael J. Schuh, PharmD, MBA, FAPhA, Ambulatory Pharmacist

Assistant Professor of Family and Palliative Medicine Assistant Professor of Pharmacy, Mayo Clinic Florida, 4500 San Pablo Road, Jacksonville, FL 32224

Email: schuh.michael@mayo.edu; Phone: 904-953-2673 specialist providers before a diagnosis can be made. In the meantime, multiple providers may prescribe multiple medications leading to polypharmacy and resulting iatrogenic symptoms which then results in more polypharmacy. ${ }^{6}$ Symptoms may not resolve no matter how many neurologic medications are prescribed because the cause is psychiatric. Both CD and SSD present with physical symptoms though CD symptoms are more likely to be neurologic and manifested as pseudoseizure or maybe tremors but will not fit any neurologic diagnosis, or if it does, may not be consistent with criteria. ${ }^{5}$ SSD patients may obsess emotionally or behaviorally over symptoms, whereas $C D$ are less obsessive or overall show less concern. $^{5}$

There are multiple models proposed as to etiology of $C D$. Predisposing factors for $\mathrm{CD}$ can be stressors or trauma. These tend to occur prior to the onset of symptoms. Child sexual abuse has been found as a cause in many studies. ${ }^{8}$ This patient had a history of opioid addiction and difficult withdrawal. This experience or the precipitating cause of the drug abuse may have contributed to this patients $C D$. This possible cause is patterned after the CD cognitive-behavioral model.

In the neurobiological model, abnormalities of grey matter regions in the limbic, orbitofrontal or anterior cingulate cortex areas of the brain are proposed. These abnormalities are proposed to cause depersonalization with abnormal sense of self-control so are interpreted as disease symptoms as they manifest physically..$^{8,9}$

In the more popular, namesake psychodynamic model, patients experience an unconscious conflict that is converted to somatic symptoms as a coping response. It is again linked to a trauma, acute or chronic, as a precipitating event to cause dissociation, possibly linked to an existing mental disorder to produce observable somatic symptoms. ${ }^{10-12}$

On the polypharmacy, PGx service, CD patients are more easily recognized due to the nature of being part of a tertiary care, destination facility that focuses on complex cases with pharmacist consult referrals commonly ordered by specialty 
providers. The same is true for other conditions where symptoms are not easily measurable. Examples are chronic serotonin syndrome, medication overuse headache and other similar difficult to diagnose conditions. The service may see multiple CD or SSD patients per month in some months. Since the service is part of a tertiary care center, patients often are referred as an additional effort to rule out medication causes, not easy to objectively measure, for symptoms since these patients have already seen multiple medical specialists to no avail of a conclusive diagnosis

It is important for clinical pharmacists to recognize SSDs and similar psychiatric disorders so focus may be directed toward elimination of polypharmacy rather than toward therapeutics that may add to polypharmacy. Clues one may be dealing with SSDs could be intolerances to therapeutically and chemically different medications but result the same symptoms, an attempt by the patient to steer the clinician to specific medications that may be less suitable for the condition being treated or symptoms that do not match side effects of the medications being used. When a clinical pharmacist is confronted with these types of psychiatric disorders, it is important to recognize them so the focus may be redirected from therapeutics selection or dosing of medications that may add to polypharmacy, to the elimination of medications from the patient's regimen to decrease polypharmacy. Use of pharmacogenomics as an additional tool may help rule out the possibility of a true MRP as etiology for a patient's symptoms.

\section{Case Summary}

The patient was a 51 year old Caucasian female with a past medical history of high dose opioids with a long, difficult withdrawal period. It was after this withdrawal the patient began demonstrating symptoms of tremor when introduced to new medications for various conditions. She was referred to the polypharmacy, pharmacogenomic (PGx) service by the department of physical medicine for involuntary, myoclonic limb jerking after these symptoms began. She showed the clinician a video of this phenomenon on her phone. The physician wanted to rule out PGx as a possible etiology for symptoms. Diphenhydramine helped alleviate the jerking but did not resolve it.

In addition to myoclonic jerking, past medical history is positive for right sided flank pain associated with Lyme disease, hypertension, nephrolithiasis and past diagnosis of a chronic, central sensitivity pain syndrome for which she was inappropriately treated with high dose opioids over an extended period of time. ${ }^{13}$ Family history positive for hypertension, heart disease, and diabetes. Musculoskeletal and neurological exams performed by the physical medicine physician and neurologist were all normal. After examination the neurologist suspected a possible somatic symptom disorder.
PGx results with regard to her tremors and relation to current medications were unremarkable. (Table II) She is a rapid CYP2C19, CYP1A2 metabolizer and possibly more prone to adverse drug reactions to statins (SLCO1B1) and certain chemotherapeutic agents due to atypical TPMT and UGT1A1 variants. None of her current medications or symptoms relate to the above variant genes so PGx results cannot explain a pharmacologic etiology for her tremors. (Table II)

After visiting with the patient, analysis of her PGx report, then through the process of exclusion, the pharmacist suspected CD. Recommendation from the pharmacist was to attend the institution's 3 week pain rehabilitation clinic (PRC) that supports patients with physical and occupational therapy as well as emphasizes cognitive behavioral therapy (CBT) to learn how to non-pharmacologically manage pain and other psychological and psychiatric issues. PRC aims to provide the patient with multiple strategies to help manage pain, decrease medication burden, increase functional independence, and improve quality of life. She resisted the possibility of seeing a psychiatrist. The neurologist and physical medicine physicians agreed, and the patient was referred to the PRC. After the program, the patient was much improved, and the jerking largely abated when new medications were introduced thereafter. There is still some patient apprehension about starting new medications on the patient's part but her physical symptoms when starting new medications have largely disappeared. Diphenhydramine was no longer needed to alleviate symptoms.

\section{Discussion}

The institution where this patient was seen is a tertiary care, destination medical facility. Therefore, has many specialty, subspecialty physicians and other providers who are frequently called upon to diagnose and treat conditions and disorders local or regional providers either miss or are ill equipped to handle. This can be due to of the lack of more specialized resources or time. This practice environment leads to a higher frequency of complex patients seen with not easily recognized or diagnosed conditions. Sometimes these conditions are rare and uncommon, other times the conditions are more common but difficult to diagnose and frequently are inappropriately treated or treated for a condition the patient does not have. The polypharmacy service regularly sees patients treated inappropriately for conditions they do not have due to missed diagnoses or due to polypharmacy as a cause.

\section{Practice Implications}

Clinical and community pharmacists everywhere may see CD frequently. It is a common psychiatric condition. However, pharmacists are not usually well versed in psychiatry clinically and therefore may not recognize a referred polypharmacy or pharmacogenomics patient as a CD patient. By working collaboratively with psychiatrists, neurologists, physical medicine colleagues and using their clinical training, experience and PGx testing as an additional tool, clinical pharmacists may 
add value to patient care by assisting with diagnoses and appropriate treatment.

Conflict of Interest: The authors have no conflicts of interest or financial ties to disclose.

The opinions contained in the paper are those of the authors.

\section{References}

1. Carson AJ, Brown R, David AS, et al. Functional (conversion) neurological symptoms: research since the millennium. J Neurol Neurosurg Psychiatry. 2012;83(8):842. Epub 2012 Jun 3.

2. Ali S, Jabeen S, Pate RJ, et al. Conversion DisorderMind versus Body: A Review. Innov Clin Neurosci. 2015;12(5-6):27-33.

3. Stone J, Smyth R, Carson A, et al. Systematic review of misdiagnosis of conversion symptoms and "hysteria". BMJ. 2005;331(7523):989. doi:10.1136/bmj.38628.466898.55.

4. American Psychiatric Association. Diagnostic and Statistical Manual of Mental Disorders, Fifth Edition (DSM-5), American Psychiatric Association, Arlington 2013.

5. Hurwitz TA. Somatization and Conversion Disorder. The Canadian Journal of Psychiatry. 2004;49(3):172178. doi:10.1177/070674370404900304.

6. Olashore AA, Rukewe A. Polypharmacy among children and adolescents with psychiatric disorders in a mental referral hospital in Botswana. BMC Psychiatry. 2017;17(1):174. doi:10.1186/s12888-0171347-6.
7. Stone J, Sharpe M, Binzer M. Motor conversion symptoms and pseudoseizures: a comparison of clinical characteristics. Psychosomatics. 2004;45:492.

8. Sharpe D, Faye C. Non-epileptic seizures and child sexual abuse: a critical review of the literature. Clin Psychol Rev. 2006;26(8):1020-40. doi: 10.1016/j.cpr.2005.11.011. Epub 2006.

9. Carson AJ, Brown R, David AS, et al. UK-FNS. Functional (conversion) neurological symptoms: research since the millennium. J Neurol Neurosurg Psychiatry. 2012;83(8):842-50. doi: 10.1136/jnnp2011-301860. Epub 2012.

10. Edwards MJ, Adams RA, Brown H, et al. A Bayesian account of 'hysteria'. Brain. 2012;135(Pt 11):3495512. doi: 10.1093/brain/aws129. Epub 2012.

11. Abbey SE, Wulsin L, Levenson JL. Somatization and somatoform disorders. In: The American Psychiatric Publishing Textbook of Psychosomatic Medicine: Psychiatric Care of the Medically III, Second Edition, Levenson JL (Ed), American Psychiatric Publishing, Inc., Washington, DC 2011. p.261.

12. Stone J, Carson A, Sharpe M. Functional symptoms in neurology: management. J Neurol Neurosurg Psychiatry. 2005;76 Suppl 1(Suppl 1):i13-21. doi: 10.1136/jnnp.2004.061663.

13. Stone J, LaFrance WC Jr, Levenson JL, Sharpe M. Issues for DSM-5: Conversion disorder. Am J Psychiatry. 2010;167(6):626-7. doi: 10.1176/appi.ajp.2010.09101440.

14. Turner JA, Shortreed SM, Saunders KW, et al. Does association of opioid use with pain and function differ by fibromyalgia or widespread pain status? Pain. 2016;157(10):2208-2216. doi:10.1097/j.pain.0000000000000631. 
Table 1

Conversion Disorder (CD) Somatization Symptom Disorder (SSD) Comparison ${ }^{1}$

\begin{tabular}{|l|l|}
\hline \multicolumn{1}{|c|}{ Conversion Disorder } & \multicolumn{1}{|c|}{ Somatization Symptom Disorder } \\
\hline $\begin{array}{l}\text { Conversion disorder is a specific form of somatization in } \\
\text { which the patient presents with symptoms that are only of } \\
\text { the voluntary central nervous system }\end{array}$ & $\begin{array}{l}\text { Somatic symptoms are a psychological defense against } \\
\text { mental instability }\end{array}$ \\
\hline $\begin{array}{l}\text { Neurologic symptoms do not follow known neurologic } \\
\text { damage patterns. This indicates the neurologic presentation } \\
\text { is of how the patient conceptualizes presentation and } \\
\text { originates from patient beliefs on how neurologic symptoms } \\
\text { should manifest themselves }\end{array}$ & $\begin{array}{l}\text { Attention is directed toward the presenting symptoms, } \\
\text { and the real problem and source of mental instability is } \\
\text { blocked out or partly experienced, therefore not }\end{array}$ \\
\hline $\begin{array}{l}\text { When conversion disorder symptoms are a multisystem } \\
\text { somatoform syndrome, the primary diagnosis is somatization } \\
\text { disorder }\end{array}$ & $\begin{array}{l}\text { SSD is the more general syndrome for which conversion } \\
\text { disorder is a subset related to only a neurologic } \\
\text { presentation }\end{array}$ \\
\hline $\begin{array}{l}\text { The somatic defense, the conviction that "I am physically ill," } \\
\text { forms unconsciously and presents as a specific neurologic } \\
\text { malfunction. }\end{array}$ & $\begin{array}{l}\text { The somatic defense, the conviction that "I am physically } \\
\text { ill," forms unconsciously. }\end{array}$ \\
\hline
\end{tabular}

Based on Hurwitz, 2004

Table 2

Patient Medications and Relevant Genes

\begin{tabular}{|c|c|c|c|}
\hline \multicolumn{4}{|c|}{ Patient Medications } \\
\hline Medication & Dose & Time/frequency & Indication \\
\hline Nebivolol & $5 \mathrm{mg}$ & $1 / 2$ tab BID & Blood pressure \\
\hline Fluticasone NS & $50 \mathrm{mcg} / \mathrm{act}$ & 1 spray in each nostril once daily & Allergies \\
\hline Diphenhydramine & $25 \mathrm{mg}$ & 1 tab as needed for reaction & Jerking reaction to medications \\
\hline Ibuprofen & $200 \mathrm{mg}$ & 1 tab PRN (once every 2 weeks) & Joint pain \\
\hline \multicolumn{4}{|c|}{ Relevant Genes } \\
\hline Gene & Genotype & Phenotype & Current Medication \\
\hline CYP1A2 & $* 1 \mathrm{~F} / * 1 \mathrm{~F}$ & Rapid & Not applicable \\
\hline CYP2C9 & $* 1 / * 1$ & Normal & Ibuprofen \\
\hline CYP3A4/5 & $* 1 / * 1, * 3 / * 3$ & Normal & Not applicable \\
\hline CYP2D6 & $* 1 / * 35$ & Normal & Nebivolol \\
\hline CYP2C19 & $* 1 / * 17$ & Rapid & Not applicable \\
\hline
\end{tabular}

